\title{
Vision-based gait impairment analysis for aided diagnosis
}

\author{
Javier Ortells • María Trinidad Herrero-Ezquerro - Ramón A. Mollineda
}

Received: date / Accepted: date

\begin{abstract}
Gait is a firsthand reflection of health condition. This belief has inspired recent research efforts to automate the analysis of pathological gait, in or- 2 der to assist physicians in decision making. However, 3 most of these efforts rely on gait descriptions which are ${ }_{4}$ difficult to understand by humans, or on sensing tech- 5 nologies hardly available in ambulatory services. This 6 paper proposes a number of semantic and normalized 7 gait features computed from a single video acquired by 8 a low-cost sensor. Far from being conventional spatio- 9 temporal descriptors, features are aimed at quantifying ${ }_{10}$ gait impairment, such as gait asymmetry from several ${ }_{11}$ perspectives or falling risk. They were designed to be 12 invariant to frame rate and image size, allowing cross- ${ }_{13}$ platform comparisons. Experiments were formulated in ${ }_{14}$ terms of two databases. A well-known general-purpose ${ }_{15}$ gait dataset is used to establish normal references for ${ }_{16}$ features, while a new database, introduced in this work, ${ }_{17}$ provides samples under eight different walking styles: ${ }_{18}$ one normal and seven impaired patterns. A number of ${ }_{19}$ statistical studies were carried out to prove the sensitiv- ${ }_{20}$ ity of features at measuring the expected pathologies, ${ }_{21}$ providing enough evidence about their accuracy.

Keywords Gait impairment · video-based gait analysis $\cdot$ gait database $\cdot$ computer-aided diagnosis
\end{abstract}

Javier Ortells and Ramón A. Mollineda

Institute of New Imaging Technologies. Universitat Jaume I.

Castellón de la Plana, Spain.

E-mail: \{jortells,mollined\}@uji.es

María Trinidad Herrero-Ezquerro

Dept. of Human Anatomy \& Psychobiology. School of ${ }^{33}$ Medicine. Universidad de Murcia, Spain.

E-mail: mtherrer@um.es

\section{Introduction}

Gait is essentially determined by the coordinated action of musculoskeletal and nervous systems. This makes gait a reliable indicator to detect symptoms of worsening health caused by aging 34, physical malfunction [9], or neurodegenerative disorders. Some examples of these last ailments are Parkinson's disease [23, 25, 33, multiple sclerosis [16] and strokes [30]. In this regard, neurologists handle a number of diagnostic tests for assessing and manually scoring gait disorders, such as the Unified Parkinson's Disease Rating Scale (UPDRS) [5] or the Rating Scale for Gait Evaluation (RSGE) [17.

The potential of gait as a multifaceted source of knowledge has encouraged a number of applied research fields based on the automation of gait analysis. The vast majority of efforts have been focused on biometric recognition or video-surveillance systems 31. However, last decade has witnessed a growing interest in clinical applications of gait assessment such as rehabilitation [18], medical diagnosis [23, and detection of medical emergencies in hospital environments [22]. These results are supported by different sensors for extracting gait data, being wearable gadgets and vision-based devices those most popular. Sensors in the first group (e.g., gyroscopes, accelerometers, markers) 11,13 acquire precise information, although they can be deemed intrusive since they are usually attached to rigid segments of the human body, thus possibly causing discomfort to patients. Regarding the vision-based group, there are professional solutions from specialized companies (BTS, Vicon, NDI, etc.) also aimed at providing highly accurate motion data without requiring any contact with a sensor 1. However, they are generally costly and demand certain setting and calibration processes, hence their use tends to be restricted to more 
specialized environments. On the contrary, less sophis- 87 ticated vision devices such as Microsoft Kinect or plain 88 RGB cameras 22, 23, 25, 34 are also capable of captur- 89 ing motion at a distance, being usually cheaper, easier 90 to use and virtually ubiquitous.

It is well known that precision of gait descriptions 92 acquired by vision systems can be severely affected by a 93 number of factors that influence either the motion pat- 94 tern or the gait perception. Motion may be altered by 95 footwear, surface, mood, age, body weight, physical in- 96 juries, neurological disorders, or even by people's own 97 volition. Regarding the last, it has been noticed that 98 some patients affected by a neurological disease tend to 99 conceal motion impairments when they know that they100 are being recorded. On the other hand, factors that af-101 fect gait perception can be classified into three groupsio2 according to their sources: subject appearance, record-103 ing conditions and video quality. Appearance can be ${ }_{104}$ affected by changes in clothing, load carrying and cam- ${ }_{105}$ era viewpoint. Recording conditions depend on factors ${ }_{106}$ like background, illumination and occlusions. Finally ${ }_{107}$ video quality refers to limitations of optical sensors.

Fortunately, vision-based analysis of gait disorders ${ }_{109}^{108}$ is a type of task in which both physicians and $\mathrm{pa}^{-}{ }_{110}$ tients are equally interested in acquiring high-quality ${ }_{111}$ data. Therefore, it can be assumed a cooperative set- ${ }_{112}$ ting, where the majority of factors that can affect gait ${ }_{113}$ are avoided. For example, we can expect simple and $_{114}$ clean scenarios, possibly indoor, pleasant environmen- ${ }_{115}$ tal conditions, fixed background, steady illumination ${ }_{116}$ during recording, patients under controlled emotional ${ }_{117}$ states, tight clothes, flat shoes, no accessories, smooth $_{118}$ floor, etc. Also patients' efforts to conceal gait disor- ${ }_{119}$ ders can be mitigated by simply adding an acoustic or ${ }_{120}$ visual distracting element, such as music or a TV $14 . ._{121}$ Under such general conditions, extraction of silhouettes ${ }_{122}$ (source of information of the most popular gait models) can be performed accurately from plain videos acquired ${ }^{123}$ with any low-cost device (RGB cameras, smartphones, ${ }_{125}{ }^{124}$ Microsoft Kinect, etc.).

\subsection{Related works} come a fast-growing are this field, related works can be categorized as regard the analysis of either unaffected or impaired gait.

Concerning the first group, a number of works which ${ }_{134}$ measure spatio-temporal and kinematic parameters of $f_{35}$ gait from healthy people have been recently published.136 In 10, a wearable 2D system based on an smartphone137 fixed in a belt is proposed. The phone includes a cam-138 era which tracks two markers placed on feet to com-139 pute step lenght, width and time, gait speed and double support time. In another work 24, a simple RGB webcam is used together with markers to get kinematic gait parameters from people walking in a treadmill. Concurrently, 3D low-cost approaches have gained in popularity since Microsoft Kinect was released. For instance, in 3 and 4 a Kinect-based marker-less solution was validated against a more sophisticated system consisting of 8 IR cameras, when quantifying lower limbs motion. In a different approach [27, several machine learning models were fed with Kinect data to perform self-esteem recognition based on people's gait pattern. A comparison between a Kinect-based method and a wearable sensor-based solution is presented in 6. Accuracies of both frameworks at estimating temporal gait parameters were assessed over people belonging to two age ranges, using GAITRite as gold standard.

On the other side, manifold vision methods which delve into the analysis of impaired gait have been proposed. The work in 34 addresses the problem of discriminating two categories of pathological gait commonly seen in senior people, which are caused by leg and visual impairments respectively. Gait was represented by a PCA+LDA transformation of GEI features elicited from body patches. Experiments were performed on gait sequences of normal people wearing knee pads that restrict knee bending, and glasses that blur the sight and narrow the view field, both tools from an age simulation kit. In the case of 32 , it focuses on recognizing walking styles, including both abnormal and normal gait, based on PCA features obtained from frame-toframe optical flow data. Pathological styles were recreated by a single trained professional actor. The last two proposals prioritized recognition based on information far from human awareness, over a comprehensible characterization of gait abnormality.

Focusing on typical ailments that affect motion, many works address gait impairment associated to Parkinson's Disease (PD). In 23, authors evaluate the discriminant power of several gait parameters extracted from Kinect data, for distinguishing between PD patients treated with deep brain stimulation and control subjects. In [25], a Kinect-based approach for analyzing the movements of PD patients during rehabilitation treatment is presented, as a preliminary step towards a system suitable for home usage. Gait analysis consists simply in the estimation of gait speed and hand rigidity while subjects are walking from 3.5 to $1.5 \mathrm{~m}$ away from the Kinect. The work in 28 also delves into the use of Kinect for describing walking parameters and recognizing gait disorders in PD patients. After filtering and smoothing the signal, two gait features were estimated: step length normalized to leg length, and walk- 
ing speed. Then, they were involved in a 1-NN classifi-187 cation process. In [12, a portable solution for assessing ${ }_{188}$ Parkinsonian gait in common environments is proposed, ${ }_{189}$ based on monocular image sequences of patients wear-190 ing markers attached to knee and ankle joints. A num-191 ber of basic gait parameters, such as gait cycle time,192 stride length, walking velocity and cadence, were mea-193 sured from videos and their reliability validated againstig4 the GAITRite system. Results showed the relevance of 195 stride length and walking velocity at distinguishing $\mathrm{PD}_{196}$ before and after drug administration.

\subsection{Open issues}

After literature review, some issues are worthy of fur-202 ther consideration. On the one hand, some works ad 203 dress automatic classification of gait impairment based ${ }^{204}$ on unreadable or basic gait features. However, since ${ }^{205}$ gait disorders are generally evident to the naked eye,206 making an obvious decision between patients or healthy ${ }^{207}$ people seems to have no practical sense. At most, the ${ }^{208}$ usefulness of classification tasks would be limited to as ${ }^{209}$ sess the discriminant capacity of features (as it is made ${ }^{210}$ clear in [1). Thus, the design of features that provide ${ }^{211}$ human-friendly quantification of a visible gait disorder ${ }^{212}$ is supposed to be of much more interest for physicians than a superfluous classification process.

On the other hand, there are virtually no published ${ }^{213}$ benchmarking efforts. There exist almost as many datasets, preprocessing techniques, gait feature sets and experimental methodologies as research works. In addi $_{215}$ tion, most datasets are not publicly available. This sce- ${ }_{216}$ nario makes it hard to establish the real merits of cur- $^{-217}$ rent approaches.

\subsection{Scope and goals}

This paper introduces a semantic, vision-based charac-223 terization of gait impairment to directly assist physi-224 cians in diagnostic decisions. Instead of measuring typ-225 ical spatio-temporal parameters, a number of normal-226 ized and invariant gait features quantify impaired gait 227 patterns, such as multiple views of gait asymmetry and $\mathrm{d}_{28}$ risk of falling. Normalization makes these features an $\prod_{22}$ easy-to-interpret source of information, while the in-230 variance to recording parameters, such as frame rate231 and image resolution, provides consistency in cross-plat-232 form comparisons. In contrast to most previous efforts,233 which rely on cryptic or plain gait descriptors, or on $_{234}$ less pervasive technologies, the feature set proposed in 235 this paper could be embedded in a low-cost vision sys-236 tem (e.g. a mobile phone or a Kinect-based solution) to directly assist clinicians in quantifying gait disorders.

This paper also presents a new dataset, the INIT Gait Database, which consists of video recordings of a number of volunteers simulating different patterns of pathological gait, along with their natural walking style. It is intended to validate the effectiveness of the features at characterizing known gait disorders. This dataset is made publicly available to the research community, with the aim of encouraging future studies involving other tasks or features.

Experiments involve the new dataset and a generalpurpose gait database. The latter comprises independent regular gait samples, which were used to establish reliable neutrality baselines for all features, and to statistically verify whether the INIT samples recorded under the natural walking style fit this expectation. Afterward, the capacity of features to precisely characterize irregular gait patterns was statistically studied.

The rest of the paper is structured as follows. Section 2 establishes the fundamentals of human gait and presents the main contributions of this work: the devised video-based features and the new INIT Gait Database. Experiments are presented and discussed in Sections 3 and 4 . Finally, Section 5 provides the conclusions and some future work highlights.

\section{Theory and methods}

\subsection{Human gait}

Normal gait can be defined as a cyclic movement pattern under two main assumptions 26, 29]: i) cycles are identical, and ii) left and right limbs perform in a similar way (i.e., both halves of each cycle are symmetrical). These assumptions are normally not fully met in practice; however, they can be considered consistent expectations for most people.

A gait cycle is composed of two principal phases: stance, where a particular foot is on the ground, and swing, where this same foot is no longer in contact with the ground and it is moving forward. Start and end of these phases are determined by two main gait events: a heel strike $(H S)$ of a foot represents its first contact with the ground, initiating the stance phase, while the transition between stance and swing is produced by a toe off (TO) event, when the foot leaves the ground starting a new step. Concurrently, the other foot follows a similar dynamic pattern half a cycle after (or before). In normal gait, stance and swing phases are expected to take $62 \%$ and $38 \%$ of a regular cycle, respectively 29 . Figure1 1 illustrates this distribution, from the right limb perspective, along a full gait cycle. 


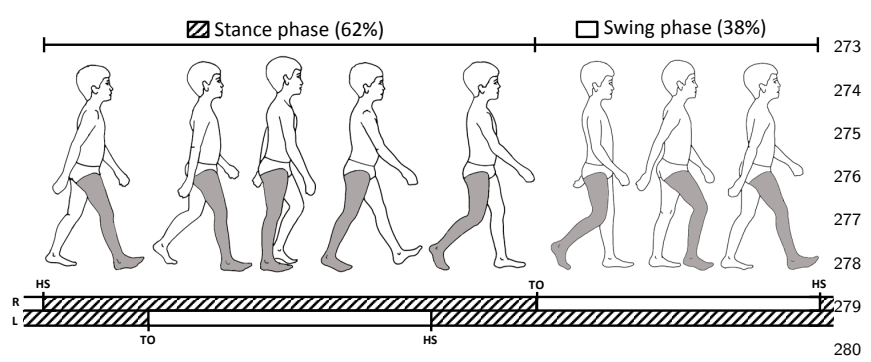

Fig. 1 Gait cycle from the right limb perspective through its $s_{281}$ phases stance and swing. Events heel strike $(H S)$ and toe off (TO) determine the start and end of these phases. The com- ${ }^{282}$ plementary stance/swing distribution for the opposite limb is ${ }^{283}$ also included in the lower part. This image is inspired in one284 from [29].

These theoretical assumptions are considered nec- ${ }^{287}$ essary conditions for normal gait, but not sufficient. ${ }^{288}$ That is, a pathological gait can potentially yield identi- ${ }^{289}$ cal symmetrical cycles that meet the $62: 38$ distribution $^{290}$ of stance and swing phases. However, gait abnormal- ${ }^{291}$ ity is generally characterized by asymmetrical patterns ${ }^{292}$ or by stance/swing imbalance. As a way of example, ${ }^{293}$ gait asymmetry has been observed in patients affected ${ }^{294}$ by PD [21] and by cerebrovascular accidents 30 . This ${ }^{295}$ paper takes advantage of such evidence to formulate $a^{296}$ comprehensible description of gait (a)symmetry.

\subsection{Data processing}

A number of video-based features have been devised to be computed from binary frames, where foreground $\left(a_{302}\right.$ silhouette) appears in white over a black background.

Henceforth, the term feature is used interchangeably ${ }_{303}$ with measure.

Given a frame from a gait video, it is binarized by $\mathrm{by}_{305}$ simple background subtraction techniques. Then, the ${ }_{306}$ silhouette is extracted as a new cropped picture keep- ${ }_{307}$ ing the absolute position of its bounding box in the $\mathrm{s}_{308}$ original frame for further calculations. Finally, all sil- ${ }_{309}$ houette images are scaled under a common height, but $_{310}$ variable widths to keep their particular aspect ratios. ${ }_{311}$

Furthermore, some of the proposed measures are computed on a silhouette-based gait representation na- ${ }_{312}$ med Gait Energy Image (GEI) 8, instead of directly using raw silhouettes. GEI can be considered the most ${ }_{313}$ popular model-free method for condensing subject's dy-314 namic and appearance. It is the mean image of a se-315 quence of normalized binary silhouettes, as illustrated $d_{36}$ in Fig. 2. To construct it, the height-scaled silhouettes are horizontally aligned by the $x$-coordinate of their ${ }_{317}$ upper-half centroids and, if needed, neutral background columns are added to both sides so as to obtain equal-318 sized images. Then, they are pixel-wise averaged. Sinces19
GEI collects information of many silhouettes, it is widely known by its robustness to silhouette defects [20]. Moreover, its way of computation guarantees the independence of feature values from recording parameters.

With the aim of obtaining gait asymmetry measurements, all features (except one related to posture) are computed separately for each lower limb. To this end, given a full sequence of silhouettes, it is split up into segments delimited by midstance/midswing poses, i.e. each segment comprises half a cycle. Two groups of segments are built taking them by turns, in such a way one group contains odd segments and the other, even ones. A representative step length is elicited from each group, such that group with the shortest (longest) step is labeled as $A(B)$. Since the ultimate goal is to assess gait asymmetry, the final correspondence between left/right limb and $A / B$ group is irrelevant.

The representative step length of a group is here given by the median of measurements from all segments belonging to it. Median was chosen due to its greater robustness to outliers as compared to the mean. This same strategy is extended to obtain the limb-dependent representative values of proposed features, except for those based on GEI. In these cases, two GEI representations are built from all silhouettes (of every segment) belonging to either $A$ or $B$ groups, respectively. Since GEI is a mean image, this approach is expected to be more reliable than choosing the median of a series of rough GEIs comprising single half-cycle data.

\subsection{Gait and posture features}

Figure 3 shows a diagram with the taxonomy of the proposed features, which have been split up into two categories: gait-based (Sect. 2.3.1) and postural (Sect.2.3.2). Regarding the gait-based category, two branches can be identified. All features listed on the left side of each one are considered primary features, since they are directly inferred from gait data. Conversely, features on the right side represent asymmetry measurements derived from corresponding primary features.

\subsubsection{Gait-based features}

Let $f$ denote a generic primary feature. Let $f_{A}$ and $f_{B}$ be the representative values of $f$ computed on $A$ and $B$ groups, respectively. From them, an $f$-based gait asymmetry measure $A_{f}$ can be defined as follows:

$$
A_{f}=\frac{\left|f_{A}-f_{B}\right|}{\max \left(f_{A}, f_{B}\right)}
$$

As observed, image of $A_{f}$ is $[0,1]$, with 0 corresponding to a perfect symmetrical gait pattern and 1 to the 


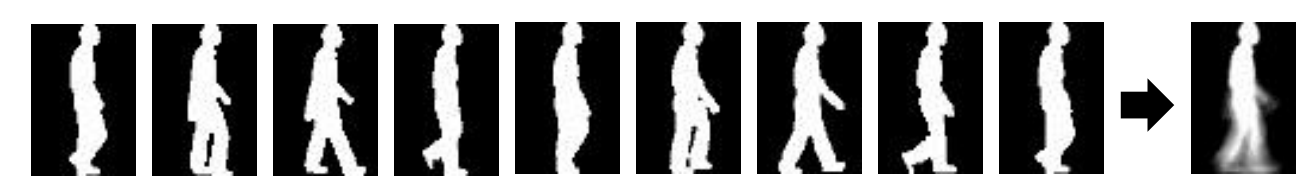

Fig. 2 Gait sequence through a series of key silhouettes, and the resulting Gait Energy Image (GEI).

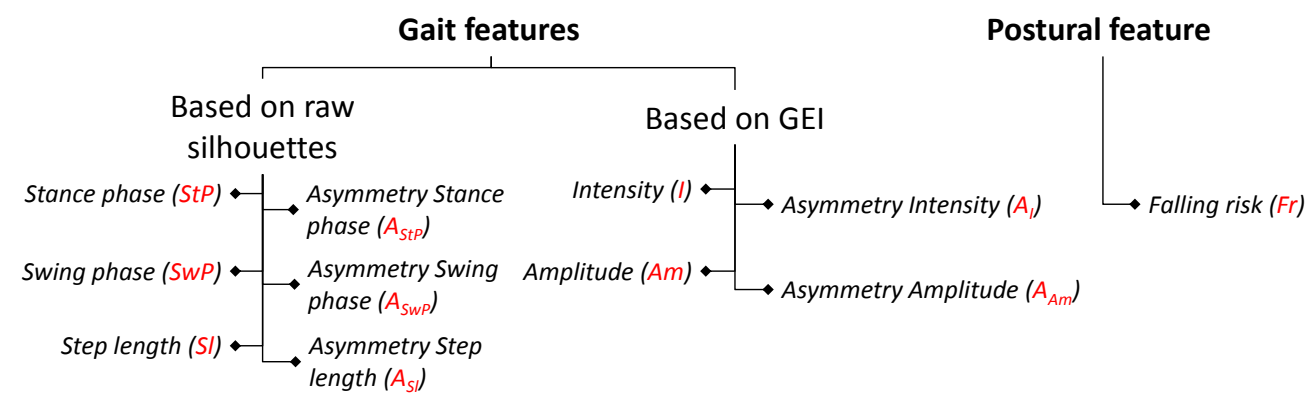

Fig. 3 Taxonomy of the proposed gait and posture features.

maximum gait asymmetry. Equation (1) can be consid-355 ered a normalized relationship between two paired mea-356 surements $\left(f_{A}, f_{B}\right)$ from a same subject, what makes357 it suitable for cross-dataset experiments. The devised 358 primary gait features $f$, from which this asymmetry359 measure is elicited, are introduced below.

As aforementioned, gait-based features are further 361 divided into two subgroups as regards the type of input362 data, which can be either the raw binary silhouettes or ${ }_{363}$ GEI representations. Within the first subgroup, three 364 primary features are proposed:

- Stance phase (StP). It estimates the relative lengtt36 of the stance phase in a gait cycle. It is formulated as ${ }^{367}$ $S t P=\frac{\text { stance }}{\text { stance }+ \text { swing }}$, where stance and swing are the $\mathrm{e}^{368}$ amounts of frames belonging to these two phases. ${ }^{369}$

- Swing phase $(S w P)$. It estimates the relative lengt $77^{\circ}$ of the swing phase in a gait cycle. It is formulated ${ }^{371}$ as $S w P=\frac{\text { swing }}{\text { stance+swing }}$, where stance and swing ${ }^{32}$ are the amounts of frames belonging to these $\mathrm{two}^{373}$ phases.

- Step length $(S l)$. It represents the distance (in ${ }^{375}$ pixels) covered by one foot in a step.

Given a particular limb, $S t P$ and $S w P$ compute the distribution over time of stance and swing phases, contrary to their common definition in literature as exclusively temporal measures. In other words, StP and $S w P$ are reformulated as the portions $\in[0,1]$ of gait cycles taken up by stance and swing phases, respectively. Note that both measures do not depend on frame rate.377

Conventionally, detection of start and end of thesesz8 phases is carried out by identifying the $\mathrm{HS}$ and $\mathrm{TO}_{379}$ events within gait cycles [7,19. Nevertheless, patholog-380 ical gait styles could entail major difficulties to obtain 381 these events. To properly deal with expected gait dis-382 orders, in this work stance phase is assumed to start383 at the moment (video frame) when distance between feet is maximum, i.e. the bounding box of the lower half of the silhouettes within a segment does not grow anymore. For its part, swing phase is deemed to start when rear leg is starting to move forward, i.e., bounding boxes begin to decrease. This method was statistically validated against a standard procedure 7$]$ by the results over high-quality neutral sequences, and no significant differences were found.

In the case of $S l$, it is generally obtained by measuring the distance between two consecutive heel strikes what, again, could be extremely inaccurate in severely affected gait patterns. Therefore, it has been inferred here by measuring the width (in pixels) of bounding box enclosing the lower part of the silhouette in the frame when stance phase starts. The use of pixel as unit of measurement in silhouettes with standardized sizes also facilitates cross-dataset comparisons.

The second subgroup comprises two other primary features based on GEI representations which, to our knowledge, are introduced for first time in this work. The proposed features are:

- Intensity $(I)$. It is defined to show the amount of movement within a GEI area:

$$
I=\frac{\sum_{p \in F} I_{p}}{|F|}
$$

where $I_{p}=1-\frac{\left|g_{p}-127.5\right|}{127.5}$ measures the motion at a foreground pixel $p$, with $g_{p}$ and $F$ being the gray level of $p$ and the set of foreground pixels, respectively. The closer to $127.5 g_{p}$ is, the higher the estimated motion (up to 1 ). That is, 127.5 would correspond to a pixel $p$ that has been background $(0)$ in half of the frames, and foreground (255) in the other 
half. This scenario can be considered of maximum ${ }_{424}$ movement, leading to $I_{p}=1$.

- Amplitude $(A m)$. It is defined to show the limbars movement's broadness:

$$
A m=\frac{|F|}{|F|+|B|},
$$

where $F$ and $B$ are the sets of foreground and background pixels, respectively, with $|F|$ and $|B|$ denot- $_{432}$ ing the cardinality of both sets.

Since these two features are intended to focus on $_{435}^{434}$ lower limb activity, GEI area was limited to the bottom $33 \%$, which encloses approximately knees and feet. ${ }^{43}$ To build $F$ and $B$, GEI pixels with gray values greater ${ }^{437}$ than or equal to 10 were considered foreground, while ${ }^{438}$ those lower than 10 were classified as background. As ${ }^{439}$ commented in Sect. 2.2. unlike in the previous three fea ${ }^{440}$ tures based on raw silhouettes, $f_{A}$ and $f_{B}$ values of each ${ }_{442}^{441}$ GEI-based $f$ are computed from two limb-dependent ${ }_{443}^{44}$ global GEIs.

\subsubsection{Postural feature}

In addition to gait-based features which characterize gait dynamics and asymmetry, a way of measuring the ${ }^{488}$ falling risk $(F r)$ is formulated by relating patient's 's49 support area and body tilt. Both parameters are com-450 puted from those frames in which feet reach the largest ${ }^{451}$ distance between them. Support area is measured from ${ }^{452}$ the toe of front foot to the heel of rear foot, while body $y^{453}$ tilt is determined by the head position on $x$-axis. For-454 mally, falling risk is defined as follows:

$$
F r=\min \left(1, \frac{\left|x_{h}-\bar{x}_{f}\right|}{w_{f} / 2}\right)
$$

where $x_{h}$ is the $x$-centroid of the head, $\bar{x}_{f}$ is the middle ${ }^{460}$ point between feet in the $x$-plane, and $w_{f}$ is the width461 of the support area. As far as we know, this proposal is462 also a novelty of this paper.

463

The minimum falling risk, $F r=0$, is reached when ${ }^{464}$ $x_{h}=\bar{x}_{f}$, that is, when head is vertically aligned with ${ }^{465}$ the center of the support area. On the contrary, the ${ }^{466}$ maximum probability of falling, $F r=1$, occurs when ${ }^{467}$ the $x$-centroid of the head coincides with, or is located ${ }^{468}$ beyond, the front limit of the support area. As in the ${ }^{669}$ silhouette-based measures defined in Sect. 2.3.1 this feature is computed once per segment. However, in this case there is no further distinction in $A$ and $B$ groups. The final $F r$ value is the median of measurements from all segments together.

\subsection{The INIT Gait Database}

The proposed INIT Gait Databas $\mathrm{C}^{\mathrm{T}}$ consists of sequences of high-quality binary silhouettes extracted from RGB videos recorded in the specialized studio LABCOM, which belongs to the audiovisual facilities of University Jaume I. Ten healthy volunteers, nine males and one female, were required to walk across a green chroma simulating several abnormal gait styles. The use of such uniform background facilitated the binarization of frames and extraction of high-quality silhouettes, thus reducing the uncertainty when evaluating the accuracy of features.

Seven impaired gait styles were simulated, in which movement of limbs and posture of the entire body were altered to some extent. They are inspired by pathological gait patterns that are characteristic of certain neurological diseases such as Parkinson. An eighth style of natural and unaffected motion has also been included. Each person was recorded twice under each gait pattern, and all sequences were acquired from a lateral view, from which limb motion and body posture can be better described. Gait styles of the INIT Gait Database are summarized below, named as in the database file structure:

nm It represents the normal gait pattern of a healthy person, which is also referred to as neutral or regular appearance in the database.

l-r0.5 It recreates a gait pattern in which right leg takes steps roughly one half shorter than left leg.

l-10.5 It recreates a gait pattern in which left leg takes steps roughly one half shorter than right leg.

fb It recreates a severely affected gait pattern in which the full body presents a number of abnormal gait symptoms: subjects walk slowly, bending the knees, and taking very short steps barely rising feet from ground (shuffling gait). Posture is also considerably modified with respect to a healthy gait style, losing the vertical position and excessively bending head and chest forwards. These symptoms are common in advanced stages of the Parkinson's disease.

a-r0.5 It recreates a gait pattern in which right arm swings approximately one half less than left arm.

a-10.5 It recreates a gait pattern in which left arm swings approximately one half less than right arm.

a-r0 It recreates a gait pattern in which right arm does not swing at all.

1 For reviewing purposes, the database can be directly downloaded from http://www.vision.uji.es/ gaitDB/INIT_GaitDB.zip (password to uncompress: "INIT_GaitDB2017UJI"). The final version will include a public website with instructions to download. 


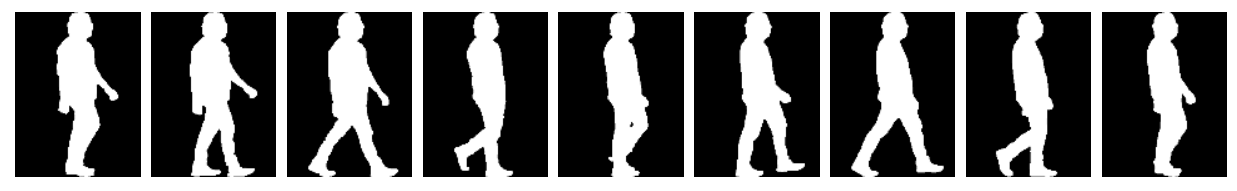

(a) nm: Normal gait pattern of a healthy person.

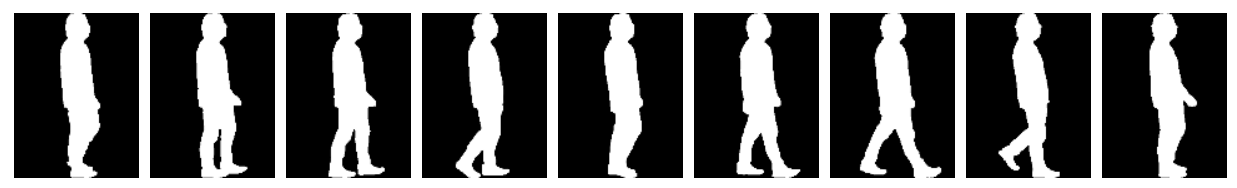

(b) 1-10.5: Left leg takes steps approximately one half shorter than right leg.

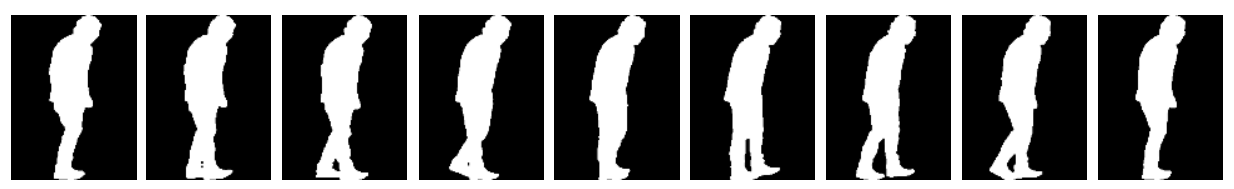

(c) fb: Severely affected gait pattern where the full body presents abnormal symptoms.

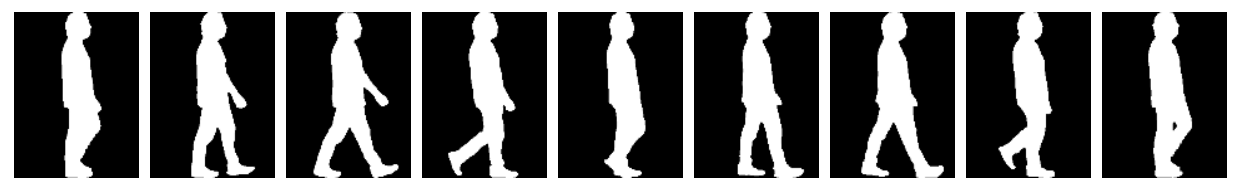

(d) a-10.5: Left arm swings approximately one half less than right arm.

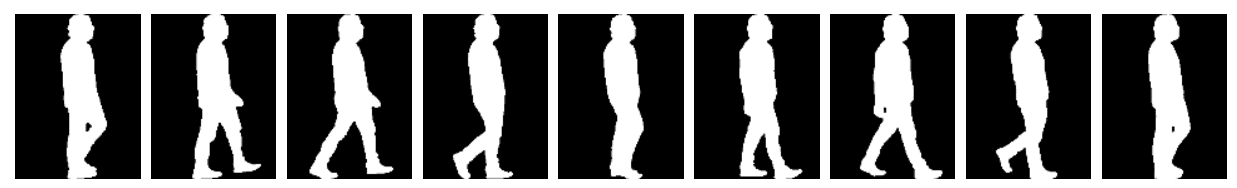

(e) a-10: Left arm does not swing at all.

Fig. 4 Samples of the different gait styles in the INIT Gait Database.

a-10 It recreates a gait pattern in which left arm does490 not swing at all.

Figure 4 shows a sample of a same subject walking ${ }^{492}$ under a) $n m$, b) $l-l 0.5$, c) $f b$, d) $a-l 0.5$ and e) $a-l 0$ gait $^{493}$ styles. The remaining three have not been included in ${ }^{494}$ the figure, since they are realizations of b), d) and e) ${ }^{495}$ styles but from the contrary limb perspective.

\section{Results}

Two experimental studies have been conducted to evaluate the sensitivity of the proposed features at characterizing both normal and impaired gait styles. First, the expected normality of the $n m$ style was assessed $_{502}$ by comparing feature values from the $\mathrm{nm}$ sequences $_{503}$ against two references, one theoretical and the other ${ }_{504}$ empirical. The relevance of proving normality of $\mathrm{nm}$ sequences lies in the confidence it provides to subsequent ${ }_{505}$ comparisons between normal and pathological styles.

This preliminary analysis was also useful to establish ${ }_{506}$ early evidence in favor of the consistency of features.507 In a second study, features were computed on several ${ }_{508}$ styles of the INIT Gait Database, to statistically verify whether features are able to reflect the anomalies recreated in the different gait patterns.

In the new INIT Gait Database (2 sequences per subject and style), each feature value used in the experiments results from averaging the two measurements obtained from both corresponding sequences of a person under analysis. Furthermore, when a primary feature $f$ is directly involved in any test, its limb-based measurements $f_{A}$ and $f_{B}$ are equally considered without any distinction.

3.1 First study: normality assessment of $\mathrm{nm}$ sequences

In this section, the expected regularity of $n m$ sequences from the INIT Gait Database is verified from both a theoretical perspective and an empirical one.

\subsubsection{Theoretical validation}

The cycle distribution between stance and swing estimated by $S t P$ and $S w P$ on $n m$ sequences was compared to their theoretical values $(62: 38)$ introduced in 
Table 1 One-sample t-tests given a known population mean for stance phase $(S t P)$ and swing phase $(S w P)$ features over the $n m$ sequences from INIT Gait Database. Symbols "o" highlight $p$-values above the significance level $\alpha=0.05$, indicating irrelevant differences between the sample and the population theoretical mean.

\begin{tabular}{ccc}
\hline & $S t P$ & $S w P$ \\
\hline & $\circ$ & $\circ$ \\
$p$-value & 0,7711 & 0,7711 \\
\hline
\end{tabular}

Section 2.1. A one-sample t-test was applied to each feature to find out whether the observed $S t P$ and $S w P_{551}$ values could have been generated by a process with the 552 mean on paper. This would allow a validation of the ${ }_{553}$ normality of $n m$ sequences assuming that $S t P$ and $S w P_{554}$ perform satisfactorily and, on the other hand, the as-555 sessment of $S t P$ and $S w P$ provided that $n m$ sequences $_{556}$ fit a normal pattern.

Table 1 summarizes the results of both parametric s. $_{58}$ tests. As can be observed, $p$-values overtake the signif-559 icance level $\alpha$, which means that the null hypothesis $s_{50}$ is not rejected and, therefore, that no relevant differ- ${ }_{561}$ ences between the theoretical mean and our samples ${ }_{562}$ have been found. This supports the assumption of nor- ${ }_{-563}$ mality of $n m$ sequences.

\subsubsection{Empirical validation}

Four gait features were used to validate the normal-s ity of the $n m$ sequences from the INIT Gait Database with respect to a collection of neutral gait sequences from the OU-ISIR Treadmill Dataset B [15]. The latter is a general-purpose gait database composed of in-569 door recordings of 68 healthy subjects from their sides70 view, wearing up to 32 clothing combinations. Due to their neutral appearance, only sequences that combines71 regular pants and full shirt were considered, which cor-572 respond to type 9 sequences according to the dataset573 nomenclature. Given a specific feature, the two popula-574 tion samples (OU-ISIR, INIT) were compared by an575 unpaired two-sample t-test, assuming equal variance.576 Under the reasonable assumption of a normal pattern577 in the selected gait sequences from OU-ISIR database,578 this test is expected to provide further evidence on thes79 normality of $\mathrm{nm}$ sequences.

The gait features included in this experiment weres81 $A_{S l}, A_{I}, A_{A m}$ and $F_{r}$. They were chosen because of ${ }_{52}$ two reasons: 1) they can be computed from sequences583 of normalized silhouettes, as provided by the OU-ISIR ${ }_{584}$ database; and 2) they were designed to be robust toss5 cross-dataset studies. Results are shown in Table 2, As586 in the theoretical validation, in none of the tests has thes87 null hypothesis been rejected. It statistically supportss8s that both samples may belong to the same population,589
Table 2 Unpaired two-sample t-tests assuming equal variances between neutral sequences from INIT Gait Database and OU-ISIR Database. Features involved are the asymmetries in step length $\left(A_{S l}\right)$, intensity $\left(A_{I}\right)$ and amplitude $\left(A_{A m}\right)$, and the fall risk factor $(F r)$. Symbols "о" highlight $p$-values above the significance level $\alpha=0.05$, indicating irrelevant differences between both samples.

\begin{tabular}{ccccc}
\hline & $A_{S l}$ & $A_{I}$ & $A_{A m}$ & $F r$ \\
\hline & $\circ$ & $\circ$ & $\circ$ & $\circ$ \\
$p$-value & 0,2957 & 0,3415 & 0,9124 & 0,1634 \\
\hline
\end{tabular}

strengthening the assumption of normality of $n m$ sequences.

Regarding the remaining features, some evidence was found which made them unsuitable to compare treadmill walking samples of Japanese people (OU-ISIR) against overground gait sequences of European subjects (INIT). For instance, 2] stressed a lower normalized step length in Asian people than in European people. Another work 26 showed significant differences in step length and stance-swing distribution between overground and treadmill locomotion, which directly affect the intensity and amplitude of leg motion. Exploratory tests with $S l, I$ and $A m$ confirmed these expected differences. In addition, StP and $S w P$ (and their corresponding asymmetries) could not be accurately computed from the out-of-context silhouettes provided by OU-ISIR, due to the fact that neither their original position in the scene nor source recordings are available.

3.2 Second study: ability of features to characterize gait anomalies

In this study, features introduced in Section 2.3 were computed on gait sequences corresponding to four styles out of the eight comprised in the INIT Gait Database. Styles involved were $n m, l-r 0.5, l-l 0.5$ and $f b$. Only those that mimic arm disorders were excluded, motivated by the belief that features formulated are not as suitable for describing arm motion as for characterizing movement in leg region. Unlike the latter, arm dynamic is largely occluded by torso; thus, appropriate features should probably weight the perceived motion by some measure of the size of trunk.

Since every subject appears walking in all styles, a number of parametric pairwise tests were applied in order to find out whether there exist statistical differences between feature values computed on normal gait patterns and those computed on each pathological style. This study has been broken down into two subsections, focusing on $n m$ vs. $f b$ and $n m$ vs. l-r0.5/l-l0.5 comparisons, respectively. 
Table 3 Paired two-sample t-tests performed on the INIT Gait Database between neutral ( $n m$ ) sequences and full body affected $(f b)$ sequences. Symbols "o" ("•") highlight $p$-values above (below) the significance level $\alpha=0.05$, indicating irrelevant (substantial) differences between samples.

\begin{tabular}{ccccccc}
\hline & $S t P$ & $S w P$ & $S l$ & $I$ & $A m$ & $F r$ \\
\hline$p$-value & $\bullet, 56 \mathrm{E}-07$ & $5,56 \mathrm{E}-07$ & $6,33 \mathrm{E}-23$ & $1,88 \mathrm{E}-13$ & $1,31 \mathrm{E}-20$ & $2,97 \mathrm{E}-07$ \\
\hline \hline & $A_{S t P}$ & $A_{S w P}$ & $A_{S l}$ & $A_{I}$ & $A_{A m}$ & \\
\hline$p$-value & $\circ$ & $\circ$ & $\bullet$ & $\bullet$ & $\bullet$ & \\
\hline
\end{tabular}

\section{2.}

3.2.1 Normal style ( $\mathrm{nm})$ versus full-body disorder style ${ }_{630}$ 632

A first analysis involved the six features that do notos3 entail asymmetries: stance phase $(S t P)$, swing phase $\mathrm{e}_{34}$ $(S w P)$, step length $(S l)$, intensity $(I)$, amplitude $(A m)_{635}$ and falling risk $(F r)$. A second analysis covered the five $e_{636}$ asymmetry-driven measures inferred from previous fea-637 tures: $A_{S t P}, A_{S w P}, A_{S l}, A_{I}$ and $A_{A m}$.

The upper half of Table 3 shows the results of paired 639 two-sample t-tests on the first group of features. As ex-640 pected, significant differences were found in the behav-641 ior of $S t P, S w P, S l, I, A m$ and $F r$. These results proves42 the sensitivity of features at reflecting the severe gait 643 impairment recreated in $f b$ samples. The second analysis comprehends the lower part of Table 3, which includes the results over the five asymmetry features. $\mathrm{No}^{644}$ statistical differences were found when computing two of them $\left(A_{S t P}, A_{S w P}\right)$, while significant changes were ${ }^{645}$ observed in $A_{S l}, A_{S t P}$ and $A_{S w P}$. Further details about ${ }^{646}$ these findings are given in Section 4 .

\subsubsection{Normal style $(\mathrm{nm})$ versus one-leg disorder styles 650 $(l-r 0.5, l-l 0.5)$

The comparison between the $n \mathrm{~m}$ style and the two one-653 leg disorder styles $(l-r 0.5, l-l 0.5)$ was based on the five $\mathrm{f}_{654}$ asymmetry features $\left(A_{S t P}, A_{S w P}, A_{S l}, A_{I}, A_{A m}\right) \operatorname{and}_{655}$ the falling risk $(F r)$. The limb-dependent primary fea- 656 tures $(S t P, S w P, S l, I, A m)$ were discarded because $\mathrm{a}_{657}$ single general value $f$ representing both limbs makes no $_{658}$ sense in asymmetrical patterns of leg motion as those $\mathrm{e}_{659}$ simulated in l-r0.5 and l-l0.5 styles.

The $t$-test results corresponding to the six involved ${ }_{661}$ features are shown in Table 4 By way of summary, in $n_{662}$ three of them $\left(A_{S l}, A_{I}, A_{A m}\right)$, significant differences 663 were found between the $n m$ and $l$-r0.5/l-l0.5 styles, 664 while the remaining three features $\left(A_{S t P}, A_{S w P}, F r\right)_{665}$ showed a statistically similar behavior when operating ${ }_{666}$ in both scenarios. Next section gives a deeper interpre-667 tation of these results.

Additionally, by way of supplementary information,669 Appendix A includes two tables with the feature values6ro measured on the INIT Gait Database styles considered in the experiments. Table 5 shows the limb-dependent values of primary features and falling risk for each style, while Table 6 reflects the values of asymmetry measures. For the sake of clearness, presented feature values are averages, together with standard deviations, over all subject measurements. Note that these values do not match with those used in the experiments, where values per person were required to perform the t-tests. As it can be seen, broad margins can be identified between domains of values from the normal style and those corresponding from pathological styles. This would allow physicians to establish reliable thresholds for assessing the existence and severity of a gait disorder.

\section{Discussion}

Results have been remarkably consistent with expectations. This can be explained by two factors that, in our opinion, have been extensively verified: 1) the welldefined gait styles included in the INIT Gait Database, and 2) the effectiveness of features at characterizing the normal and pathological gait patterns.

These two premises were first tested in the study of normality of $\mathrm{nm}$ sequences (Section 3.1), which established the consonance of the empirical relative lengths of stance/swing and their ideal values. It supports both the neutrality of the $n m$ sequences and the validity of $S t P$ and $S w P$. This study also entailed a successful cross-database comparison that proved the robustness of features to different video settings. As commented, it makes possible to directly compute gait features from videos acquired by heterogeneous devices.

As regards the second study (Section 3.2), Table 3 shows consistent behaviors of the primary features when coping with two quite dissimilar symmetrical styles such as $n m$ and $f b$. This is a relevant finding since the $f b$ style is a heavily affected gait pattern that involves extra complexity to be analyzed. In particular, the greatest differences were obtained in step length $(S l)$, amplitude $(A m)$ and intensity $(I)$ of leg motion (their null hypotheses of equal means were rejected by larger margins). As regards $F r$, it was clearly affected by the 
Table 4 Paired two-sample t-tests performed on the INIT Gait Database between neutral ( $\mathrm{nm}$ ) sequences and right leg half motion (l-r0.5) or left leg half motion (l-l0.5) sequences. Symbols "०" ("•") highlight p-values above (below) the significance level $\alpha=0.05$, indicating irrelevant (substantial) differences between samples.

\begin{tabular}{cccccccc}
\hline & & $A_{S t P}$ & $A_{S w P}$ & $A_{S l}$ & $A_{I}$ & $A_{A m}$ & $F r$ \\
\hline \multirow{2}{*}{$n m$ vs. $l-r 0.5$} & & $\circ$ & $\circ$ & $\bullet$ & $\bullet$ & $\bullet$ & $\circ$ \\
& $p$-value & 0,5269 & 0,6510 & $1,87 \mathrm{E}-06$ & 0,0024 & $5,81 \mathrm{E}-06$ & 0,1611 \\
\hline \multirow{2}{*}{$n m$ vs. l-l0.5 } & $p$-value & 0,7398 & 0,7942 & $1,29 \mathrm{E}-05$ & 0,0026 & $7,94 \mathrm{E}-06$ & 0,7514 \\
\hline
\end{tabular}

hunched posture reflected by $f b$ style, as well as by its\$15 shorter steps which produce a narrow support area. $\quad 716$

Concerning the asymmetry measures from the lower part of Table 3, no statistical differences were found when computing $A_{S t P}, A_{S w P}$. This illustrates that any ${ }^{71}$ underlying alteration in stance/swing portions within the gait cycles takes place equally in both limbs, what ${ }^{718}$ effectively occurs in $f b$ style as compared to normal gait $(n m)$, leading to similar asymmetry values. It $\operatorname{can}^{720}$ be easily corroborated checking Table 5 Conversely, ${ }_{722}$ statistical differences were found on $A_{S l}, A_{I}$ and $A_{A m}{ }_{723}$ However, a closer look at their corresponding mean results in Table 6 (columns 3-5; rows 1 and 4 ) reveals very ${ }^{724}$ low asymmetry values in both $n m$ and $f b$ styles: $\leq 0.1^{725}$ in the range $[0,1]$. This behavior is explained by the greater impact of differences between $S l, I$ and $A$ mea- ${ }^{727}$ surements on both limbs (columns $A, B$ from Table $5{ }^{728}$ in the computation of $f b$ asymmetries. That is, the rel- ${ }^{229}$ ative nature of Eq. 1 stresses the influence of a given ${ }^{730}$ discrepancy when it comes from smaller magnitudes. ${ }^{731}$ The fact that such slight differences in these $n m$ and ${ }^{732}$ $f b$ asymmetry features were deemed significant by the ${ }^{733}$ statistical tests, proves them as a rigorous and reliable ${ }_{735}^{734}$ validation method.

Concurrently, asymmetry features were also very ${ }_{737}$ precise at measuring the one-half shorter step repro- ${ }_{738}$ duced by one of the legs (Table 4), a disorder that sub- ${ }_{739}$ stantially affects the symmetry of step length $\left(A_{S l}\right)_{740}$ as well as of intensity $\left(A_{I}\right)$ and amplitude $\left(A_{A m}\right) . \mathrm{As}_{741}$ shown in the table, the null hypotheses (of equal means) ${ }_{742}$ associated to their corresponding paired two-sample $t_{t_{743}}$ tests were rejected by very large margins. Nevertheless ${ }_{744}$ contrary to what might seem logical at first, a shorter ${ }_{745}$ step had no impact on stance/swing asymmetry mea- ${ }_{746}$ sures $\left(A_{S t P}, A_{S w P}\right)$. That is, a shorter step does not ${ }_{477}$ alter the portions of a gait cycle taken up by stance ${ }_{748}$ and swing stages in comparison to normal gait, as $\mathrm{re}_{749}$ flected by Table 5. Finally, no significant difference was ${ }_{50}$ found in $\mathrm{Fr}$ computation. This is also in agreement with expectations, since one-leg disorder is not supposed $\mathrm{to}_{752}$ influence subject's posture nor the support area ( hich $_{53}$ is determined by the leg with normal motion).

It is worth recalling that all measures (except $S l)_{755}$ range from 0 to 1 , what can be directly understood 756 by physicians. This fact makes them semantic, easy-tointerpret features.

\section{Conclusions}

This work proposes a readable and robust characterization of common gait and posture disorders, which consists in a number of video-based gait features. They are intended to provide normalized and invariant information when gait is being used to diagnose health condition, for instance, in primary health care for elderly people or in Parkinson's disease. Moreover, a new gait database including normal and impaired gait videos is introduced in this paper, with the object of proving the suitability of features. This dataset, named INIT Gait Database, has been made publicly available to the research community, aiming at fostering future studies about gait measurement.

A first study was conducted to test both consistency of features and neutrality of those gait samples from the new database recorded under the normal pattern. On the one hand, estimations of the relative lengths of stance and swing phases in normal gait samples were compared against their expected ideal values. On the other hand, behavior of features was analyzed when performing on normal gait samples from both the new database and a well-known general-purpose gait dataset. In a second study, sensitivity of features to reflect the impaired gait styles recreated in the new database was also assessed.

Experimental results, all of them supported by statistical tests, proved the reliability of the proposed features. In the first study, their values were in statistical agreement with their theoretical expectations and with each other when they were computed on the two independent collections of normal gait samples. This also provided strong evidence in favor of the validity of the new database. The second study showed the accuracy of features at measuring and describing different walking styles.

By way of conclusion, some promising directions for future research are suggested next. First, this paper has not delved into effective ways of characterizing arm motion. As aforementioned, arm dynamic is heavily over- 
lapped by torso, mainly in binary silhouette images.815 Any satisfactory solution to this problem should con-816 sider the extent of overlapping. To tackle this open mat- ${ }^{817}$ ter, the INIT Gait Database includes sequences where ${ }_{819}^{818}$ upper limb motion is affected at different degrees. Sec- ${ }_{-820}$ ond, from an applied point of view, the proposed fea-821 tures should be evaluated in truly impaired gait sam- ${ }^{822}$ ples, for example, from patients of Parkinson's disease. ${ }^{823}$ Our immediate goal is to work in this direction. $\mathrm{Fi}_{-825}{ }^{224}$ nally, we believe that semantic and invariant gait fea-826 tures like those proposed in this paper, along with the $e^{27}$ ease of gathering gait videos from ubiquitous simple ${ }^{828}$ devices, open the door to the development of low-cost ${ }_{830}^{829}$ vision systems that can potentially be used in ambula-831 tory services.

Acknowledgements This work has been supported by grants P1-1B2015-74 and PREDOC/2012/05 from Univ. Jaume I, and TIN2013-46522-P from Spanish Ministry of Economy and Competitiveness. Authors would also like to thank the staff ${ }_{838}^{837}$ at Communication Sciences Laboratory (LABCOM) of Univ ${ }_{839}^{838}$ Jaume I for their help in using these facilities.

\section{References}

1. Altilio, R., Paoloni, M., Panella, M.: Selection of clinicals45 features for pattern recognition applied to gait analysis.846 Medical \& Biological Engineering \& Computing 55(4),847 685-695 (2017)

2. Chen, W.L., O'Connor, J.J., Radin, E.L.: A comparison of the gaits of chinese and caucasian women with par-850 ticular reference to their heelstrike transients. Clinical ${ }_{851}$ Biomechanics 18(3), 207-213 (2003)

3. Eltoukhy, M., Kuenze, C., Oh, J., Wooten, S., Signorile ${ }^{852}$ J.: Kinect-based assessment of lower limb kinematics and ${ }_{854}$ dynamic postural control during the star excursion bal- ${ }_{855}^{854}$ ance test. Gait \& posture 58, 421-427 (2017)

4. Eltoukhy, M., Oh, J., Kuenze, C., Signorile, J.: Improved ${ }^{856}$ kinect-based spatiotemporal and kinematic treadmill gait ${ }_{858}^{857}$ assessment. Gait \& posture 51, 77-83 (2017)

5. Goetz, C.G., Tilley, B.C., Shaftman, S.R., et al.: Move- ${ }^{859}$ ment disorder society-sponsored revision of the Unified ${ }^{860}$ Parkinson's Disease Rating Scale (MDS-UPDRS): Scale ${ }^{861}$ presentation and clinimetric testing results. Movement ${ }^{862}$ Disorders 23(15), 2129-2170 (2008)

6. González, I., López-Nava, I.H., Fontecha, J., et al.: Com- ${ }^{864}$ parison between passive vision-based system and a wear- ${ }^{865}$ able inertial-based system for estimating temporal gait $^{866}$ parameters related to the GAITRite electronic walkway. ${ }^{867}$ Journal of Biomedical Informatics 62(C), 210-223 (2016) ${ }^{868}$

7. González, I., Nieto-Hidalgo, M., Mora, J., et al.: A Dual ${ }^{869}$ Approach for Quantitative Gait Analysis Based on Vision ${ }^{870}$ and Wearable Pressure Systems, LNCS, vol. 9455, pp..$^{871}$ 206-218 (2015)

8. Han, J., Bhanu, B.: Individual recognition using Gait En-873 ergy Image. IEEE Transactions on Pattern Analysis and874 Machine Intelligence 28(2), 316-322 (2006)

9. Hase, K.: A computer simulation study on the causalsz6 relationship between walking and physical malfunctions877 in older adults. Anthropological science: journal of thes78 Anthropological Society of Nippon 116(2), 95-104 (2008)879
10. Kim, A., Kim, J., Rietdyk, S., Ziaie, B.: A wearable smartphone-enabled camera-based system for gait assessment. Gait \& posture 42(2), 138-144 (2015)

11. Lau, H.Y., Tong, K.Y., Zhu, H.: Support vector machine for classification of walking conditions using miniature kinematic sensors. Medical \& Biological Engineering \& Computing 46(6), 563-573 (2008)

12. Lin, S.H., Chen, S.W., Lo, Y.C., et al.: Quantitative measurement of parkinsonian gait from walking in monocular image sequences using a centroid tracking algorithm. Medical \& Biological Engineering \& Computing 54(2), 485-496 (2016)

13. López-Nava, I.H., Muñoz-Meléndez, A., Pérez-SanPablo, A.I., et al.: Estimation of temporal gait parameters using bayesian models on acceleration signals. Computer Methods in Biomechanics and Biomedical Engineering 19(4), 396-403 (2015)

14. Ma, H.I., Hwang, W.J., Lin, K.C.: The effects of two different auditory stimuli on functional arm movement in persons with Parkinson's disease: a dual-task paradigm. Clinical Rehabilitation 23(3), 229-237 (2009)

15. Makihara, Y., Mannami, H., Tsuji, A., et al.: The OUISIR Gait Database comprising the treadmill dataset. IPSJ Transactions on Computer Vision and Applications 4, 53-62 (2012)

16. Martin, C.L., Phillips, B.A., Kilpatrick, T.J., et al.: Gait and balance impairment in early multiple sclerosis in the absence of clinical disability. Multiple sclerosis 12(5), 620-628 (2006)

17. Martínez-Martín, P., García-Urra, D., del Ser-Quijano, T., et al.: A new clinical tool for gait evaluation in Parkinson's disease. Clinical neuropharmacology 20(3), 183194 (1997)

18. Mun, K.R., Lim, S.B., Guo, Z., et al.: Biomechanical effects of body weight support with a novel robotic walker for over-ground gait rehabilitation. Medical \& Biological Engineering \& Computing 55(2), 315-326 (2017)

19. Nieto-Hidalgo, M., Ferrández-Pastor, F.J., J, V.S.R., et al.: A vision based proposal for classification of normal and abnormal gait using RGB camera. Journal of biomedical informatics 63, 82-89 (2016)

20. Ortells, J., Mollineda, R.A., Mederos, B., et al.: Gait recognition from corrupted silhouettes: A robust statistical approach. Machine Vision and Applications 28(1), 15-33 (2017)

21. Plotnik, M., Giladi, N., Balash, Y., et al.: Is freezing of gait in Parkinson's disease related to asymmetric motor function? Annals of neurology 57(5), 656-663 (2005)

22. Raheja, J.L., Chaudhary, A., Nandhini, K., et al.: Preconsultation help necessity detection based on gait recognition. Signal, Image and Video Processing 9(6), 13571363 (2015)

23. Rocha, A.P., Choupina, H., Fernandes, J.M., et al.: Kinect v2 based system for Parkinson's disease assessment. In: 37th Annual International Conference of the IEEE Engineering in Medicine and Biology Society (EMBC'15), pp. 1279-1282 (2015)

24. Saner, R.J., Washabaugh, E.P., Krishnan, C.: Reliable sagittal plane kinematic gait assessments are feasible using low-cost webcam technology. Gait \& Posture 56, 19-23 (2017)

25. Spasojević, S., Santos-Victor, J., Ilić, T., et al.: A VisionBased System for Movement Analysis in Medical Applications: The Example of Parkinson Disease, LNCS, vol. 9163, pp. 424-434 (2015) 


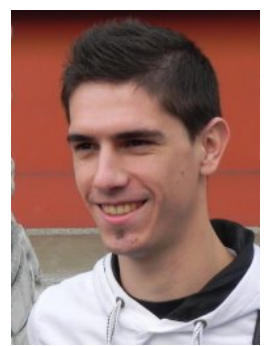

Javier Ortells has recently received a $\mathrm{PhD}$ in Computer Science from the University Jaume I, Spain. His thesis addressed gait analysis for both biometric recognition and medical diagnosis purposes.

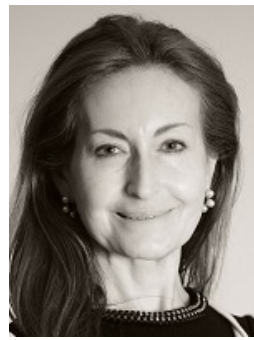

M. Trinidad Herrero-Ezquerro has a $\mathrm{PhD}$ in Medicine and Surgery from the University of Navarra, Spain. She works as a full professor at the University of Murcia, Spain, and she is currently the Director of the Institute for Research on Aging, at the University of Murcia. Her main research interests are clinical and experimental neuroscience, movement disorders, and neurodegenerative diseases.

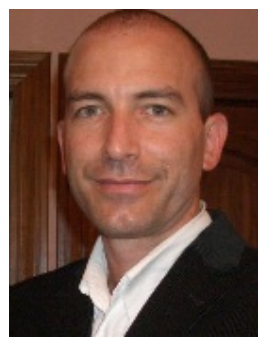

Ramón A. Mollineda has a $\mathrm{PhD}$ in Computer Science from the Polytechnic University of Valencia, Spain. He is currently an Associate Professor in the Department of Computer Languages and Systems at the University Jaume I, Spain. His research interests are machine learning, computer vision, biometrics, and gait analysis 


\section{A Feature values from the INIT Gait Database}

Table 5 Means and standard deviations of primary features, computed over all subjects for each gait style in the INIT Gait Database. Values are sorted in such a way that $A$ columns always correspond to the leg with a lower $S l$ in each style.

\begin{tabular}{rrrrrrrrrrrrrr}
\hline & \multicolumn{3}{c}{$S t P$} & \multicolumn{2}{c}{$S w P$} & \multicolumn{2}{c}{$S l$} & \multicolumn{1}{c}{$I$} & \multicolumn{3}{c}{$A m$} \\
& \multicolumn{1}{c}{$\mathrm{A}$} & \multicolumn{1}{c}{$\mathrm{B}$} & \multicolumn{1}{c}{$\mathrm{A}$} & $\mathrm{B}$ & $\mathrm{A}$ & $\mathrm{B}$ & $\mathrm{A}$ & $\mathrm{B}$ & $\mathrm{A}$ & $\mathrm{B}$ & \\
\hline \multirow{2}{*}{$n m$} & $0.62 \pm$ & $0.61 \pm$ & $0.38 \pm$ & $0.39 \pm$ & $106.13 \pm$ & $108.45 \pm$ & $0.65 \pm$ & $0.66 \pm$ & $0.55 \pm$ & $0.56 \pm$ & $0.07 \pm$ \\
& 0.03 & 0.03 & 0.03 & 0.03 & 6.75 & 6.42 & 0.01 & 0.02 & 0.04 & 0.04 & 0.04 \\
\hline \multirow{2}{*}{$l-r 0.5$} & $0.61 \pm$ & $0.61 \pm$ & $0.39 \pm$ & $0.39 \pm$ & $72.50 \pm$ & $104.30 \pm$ & $0.54 \pm$ & $0.66 \pm$ & $0.40 \pm$ & $0.54 \pm$ & $0.10 \pm$ \\
& 0.03 & 0.04 & 0.03 & 0.04 & 12.81 & 11.29 & 0.09 & 0.02 & 0.06 & 0.06 & 0.06 & 0.06 \\
& $0.63 \pm$ & $0.62 \pm$ & $0.37 \pm$ & $0.38 \pm$ & $70.80 \pm$ & $103.25 \pm$ & $0.51 \pm$ & $0.67 \pm$ & $0.37 \pm$ & $0.55 \pm$ & $0.08 \pm$ \\
\multirow{2}{*}{$l-l 0.5$} & 0.04 & 0.05 & 0.04 & 0.05 & 14.37 & 7.04 & 0.11 & 0.02 & 0.06 & 0.03 & 0.04 \\
\hline \multirow{2}{*}{$f b$} & $0.71 \pm$ & $0.70 \pm$ & $0.29 \pm$ & $0.30 \pm$ & $60.38 \pm$ & $65.03 \pm$ & $0.36 \pm$ & $0.40 \pm$ & $0.32 \pm$ & $0.34 \pm$ & $0.85 \pm$ \\
& 0.06 & 0.06 & 0.06 & 0.06 & 5.31 & 7.09 & 0.06 & 0.08 & 0.03 & 0.04 & 0.16 \\
\hline
\end{tabular}

Table 6 Means and standard deviations of asymmetry features, computed over all subjects for each gait style in the INIT Gait Database.

\begin{tabular}{rrrrrr}
\hline & $A_{S t P}$ & $A_{S w P}$ & $A_{S l}$ & \multicolumn{1}{c}{$A_{I}$} & $A_{A m}$ \\
\hline \multirow{2}{*}{$n m$} & $0.03 \pm$ & $0.05 \pm$ & $0.02 \pm$ & $0.03 \pm$ & $0.04 \pm$ \\
& 0.01 & 0.02 & 0.01 & 0.01 & 0.03 \\
\hline \multirow{2}{*}{$l-r 0.5$} & $0.04 \pm$ & $0.06 \pm$ & $0.30 \pm$ & $0.18 \pm$ & $0.27 \pm$ \\
& 0.02 & 0.02 & 0.09 & 0.12 & 0.08 \\
\hline \multirow{2}{*}{$l-l 0.5$} & $0.04 \pm$ & $0.06 \pm$ & $0.32 \pm$ & $0.24 \pm$ & $0.32 \pm$ \\
& 0.03 & 0.04 & 0.11 & 0.16 & 0.09 \\
\hline \multirow{2}{*}{$f b$} & $0.02 \pm$ & $0.05 \pm$ & $0.07 \pm$ & $0.10 \pm$ & $0.08 \pm$ \\
& 0.02 & 0.04 & 0.04 & 0.06 & 0.03 \\
\hline
\end{tabular}

